\title{
Statistical Adaptive Metric Learning in Visual Action Feature Set Recognition
}

\author{
Shuanglu Dai ${ }^{\mathrm{a}, *}$, Hong Man ${ }^{\mathrm{a}}$ \\ ${ }^{a}$ Department of Electrical and Computer Engineering, Stevens Institute of Technology, 1 Castle Point \\ on Hudson, Hoboken, New Jersey, NJ07030, U.S.A.
}

\begin{abstract}
Great variances in visual features often present significant challenges in human action recognitions. To address this common problem, this paper proposes a statistical adaptive metric learning (SAML) method by exploring various selections and combinations of multiple statistics in a unified metric learning framework. Most statistics have certain advantages in specific controlled environments, and systematic selections and combinations can adapt them to more realistic "in the wild" scenarios. In the proposed method, multiple statistics, include means, covariance matrices and Gaussian distributions, are explicitly mapped or generated in the Riemannian manifolds. Typically, d-dimensional mean vectors in $R^{d}$ are mapped to a $R^{d \times d}$ space of symmetric positive definite (SPD) matrices $S_{y m}^{+}$. Subsequently, by embedding the heterogeneous manifolds in their tangent Hilbert space, subspaces combination with minimal deviation is selected from multiple statistics. Then Mahalanobis metrics are introduced to map them back into the Euclidean space. Unified optimizations are finally performed based on the Euclidean distances. In the proposed method, subspaces with smaller deviations are selected before metric learning. Therefore, by exploring different metric combinations, the final learning is more representative and effective than exhaustively learning from all the hybrid metrics. Experimental evaluations are conducted on human action recognitions in both static and dynamic scenarios. Promising results demonstrate that the proposed method performs effectively for human action recognitions in the wild.
\end{abstract}

Keywords: Feature Set Classification; Hybrid statistic modeling; Metric Learning; Manifolds selection

\section{Introduction}

Set classification has been studied within computer vision communities for a long period of time. In gait recognition, for example, frame by frame static features of a certain object are considered as a feature set. Similarly in human action recognition, spatialtemporal features uniformly extracted from frames of an action atom are considered as a feature set [1]. In addition, image sets have been commonly used in face recognitions

*Corresponding author: sdai1@stevens.edu

Preprint submitted to Journal of LATEX Templates

February 20, 2016 
[2, 3. The task of feature set classification is to classify an input feature set to one of the sets in the training gallery [4. Compared to image sets, feature sets are more diverse. They can not be easily assumed to follow certain distribution or lie in some 10 scale and affine invariant linear subspace. One of the effective techniques handling such problem is by using statistical representations to substitute the original feature samples. For action recognition in the wild scenarios, combinations of statistics from lower-order to higher-order have shown promising representation capabilities, while how to combine these multiple statistics in a near optimal way remains a technical challenge [4, 5].

15 In general, three types of statistics have been commonly applied on set modeling, i.e. sample-based statistics (SAS) 6, 7, 8, 9, subspace-based statistics (SUS) [10, 11, 12, 13, 14, 15, 16] and distribution-based statistics (DIS) 22, 3. Utilizing affine transformation and centroid of samples, sample-based statistics represent d-dimension feature sets with first-order statistics in the $R^{d}$ space. A great advantage of SAS is that 20 samples are considered as vectors so the nearest neighbor (NN) classification can be easily implemented with unified distance measures. But sample tests performed at every individual sample are often computationally expensive. Well-known sample-based methods include Minimum Mean Discrepancy (MMD) 17, Affine (Convex) Hull based Image Set Distance (AHISD, CHISD) [18, Set-to-Set Distance Metric Learning (SSDML) 9] and

25 Information Theoretic Metric Learning (ITML) 19. Differing from sample-based statistics, subspace-based statistics analyze sets lying on a specific Riemannian manifold. By learning the kernel functions or statistical metrics, the subspaces are projected back to the Euclidean spaces. The distance measures from the Riemannian manifold to the Euclidean space is often considered as the true geodesic distances, which lie in a Hilbert

30 space [20. Distance discriminant functions are then performed on the Hilbert space, and recognitions can finally be achieved by using Nearest Neighbors (NNs) method. Secondorder statistics based methods have better representation of the data, but it is hard to design a discriminant function with a unified distance measure for the manifolds. Typical subspace-based methods include Mutual Subspace Method (MSM) [11, Discriminant

35 Canonical Correlations (DCC) 10, Manifold Discriminant Analysis (MDA) 14, Grassmann Discriminant analysis (GDA) 13], Covariance Discriminative Learning (CDL) [15], Localized Multi-Kernel Metric Learning (LMKML) [16] etc. Distribution based statistics model each sample in the feature set with a distribution, which can be expressed as an expansion of the Riemannian manifold from the 2nd-order statistic space $S_{y m}^{+}$to $S y m_{d+1}^{+}$.

40 Such methods are often with 3rd-order statistics and may lead to complex parametric distribution comparison. Typical examples include Single Gaussian Models (SGM) 2], Gaussian Mixture Models (GMM) 3] and kernel version of ITML with DIS-based set model (DIS-ITML). Although 3rd-order statistics model sets with more consolidated representations, the hypothesis tests often require significant amount of computation in 45 distribution comparisons.

More adaptive forms of set modeling methods have been proposed by combining multiple statistical metrics in certain heuristic ways. Some of the recent hybrid statistical models include Projection Metric Learning (PML) on Grassmann manifold and hybrid Euclidean-and-Riemannian Metric Learning (HERML) [21. The main idea of multiple

50 statistics combination is to project measurements from multiple heterogeneous spaces into high-dimensional Hilbert spaces. The key issue then becomes the learning of unified discriminant functions from the training sets. Due to the simplicity of the subspace mapping, discriminant functions can frequently be found from single statistics. For instance, 
Set-to-Set Distance Metric Learning (SSDML) learns a proper metric between pairs of ${ }_{55}$ single vectors in Euclidean space to obtain more accurate set-to-set affine hull based distance for classification; Localized Multi-Kernel Metric Learning (LMKML) maps the 3rd-order statistics into Euclidean spaces by learning a unified metric discriminant function through reproducing kernel Hilbert spaces (RKHS). While hybrid multiple statistics perform well in realistic outdoor scenarios, but their unified discriminant functions are ${ }_{60}$ often more difficult to design. Addressing the subspace discrimination problems, Shao et al. proposed a kernelized multiview projection (KMP) for action feature set recognition. KMP discriminatively assigns weights to multiple kernelized sets with a single feature to achieve a low-dimensional subspace. However, weighting kernels directly in the linear subspaces is not an optimal way for learning kernelized sets with multiple features in

65 different scales. 22 .

Aiming at classification for sets with multiple features, this paper proposes an adaptive subspaces analysis method for learning hybrid statistical metrics. The analyzed single or multiple statistics can be used to classify sets through various feature combinations in different scales. Inspired by the discriminant function design in the second-order

70 based methods, LogDet divergence is introduced as a unified discriminant function for our metric learning. With this discriminant function, our method effectively unifies different statistics into a common measurement. Thus nearest neighbor method can be easily performed for classification. The whole process of modeling and learning consists of several steps. Firstly, heterogeneous statistics including mean, covariance matrix

75 and Gaussian distribution are introduced to project data into high-dimensional Hilbert spaces. Typically, d-dimensional mean vectors represent samples from $R^{d}$ to $S y m_{d}^{+}$expanded by a Point-to-Set projection; covariance matrices lie in Riemannian manifold $S_{d} m_{d}^{+}$and multivariate Gaussian distributions expand the second order statistics into Riemannian manifold $S y m_{d+1}^{+}$expressed by relative entropy. Secondly, by embedding

so the heterogeneous spaces into high-dimensional Hilbert spaces, the Mahalanobis distance is introduced as our discriminant metric. Then, the Hilbert spaces selection is conducted based on the minimum Hilbert subspaces. The hybrid statistics are then reduced to single or multiple statistics combination. Finally, with LogDet divergence that maps all the Hilbert space points into $R^{d}$, a constrained kernel learning is performed. Recognitions are mainly conducted on video sequences in both static and dynamic scenarios using spatial image features such as edges, SIFT, HOG, and texture features.

\section{Statistical Feature Set Modeling}

\subsection{Statistics and Subspaces Embedding}

\subsubsection{Data Statistics}

90 Let $X=\left[X_{1}, \ldots, X_{N}\right]$ denotes the training set formed by $N$ feature sets, where $X_{i}=$ $\left[x_{1}, x_{2}, \ldots, x_{M}\right] \in R^{n_{i} \times d}$ indicates the $\mathrm{i}$-th feature set, $1 \leq i \leq N$, and $n_{i}$ is the number of samples in this set. It is known that the kernel function is always defined by firstly mapping the original features to a high dimensional Hilbert space, that is $\phi: R^{d} \rightarrow F$ or Sym $^{+} \rightarrow F$, and then calculating the dot product of high dimensional statistics $\Phi_{i}$ and

${ }_{95} \Phi_{j}$ in the new space. Considering $\phi$ as an explicit mapping with the statistical kernels, $\Phi_{i}^{r}$ denotes the high dimensional feature of r-th statistics extracted from the feature set $X_{i}$. Here, $1<r<R$ and $\mathrm{R}$ is the number of statistics being used. 
We uniformly map feature set $X_{i}, 1 \leq i \leq N$ with following three statistics: samplebased, subspace-based and distribution-based statistics.

Sample-based statistics (SAS): Supported by Bregman divergence, mean vector is considered as one of the important properties describing the probability distributions. It is often used to measure the central tendency of set of samples. Given sample $x_{k} \in X_{i}$, $1 \leq k \leq M$, the mean vector $\mu_{i}$ of $X_{i}$ is computed as: $u_{i}=\frac{1}{M} \sum_{k=1}^{M} x_{k}$.

Subspace-based statistics (SUS): Within subspaces derived from eigen-decomposition, set variances are influenced by covariant matrix. Given sample $x_{k} \in X_{i}, 1 \leq k \leq M$, the covariant matrix $C_{i}$ of $X_{i}$ is computed as: $C_{i}=\frac{1}{M-1} \sum_{k=1}^{M}\left(x_{k}-\mu_{i}\right)\left(x_{k}-\mu_{i}\right)^{T}$.

Distribution-based statistics (DIS): Gaussian distribution is a very commonly occurring probability distribution, which is a continuous distribution with the maximum entropy for a given mean and variance. Therefore, the d-dimensional distribution of set

${ }_{110} X_{i}$ is modeled as a Single Gaussian Model (SGM) with an estimated d-dimensional mean vector $\widehat{m}_{k, i}, 1 \leq k \leq d$ and a covariance matrix $\widehat{C}_{i}: x \sim N\left(\widehat{m}_{i}, \widehat{C}_{i}\right)$

\subsubsection{Subspaces Embedding and Canonical Correlation-based Selection}

A point $\mu$ in the Euclidean space $R^{d}$ can be mapped into a symmetrical positive definite matrix $\left\{\mu \mu^{T}\left|R^{d \times d},\right| \mu \mu^{T} \mid>0\right\} \in S y m_{d}^{+}$. For DIS, the space of Gaussian distribution 115 is able to be embedded into a Riemannian manifold $S y m_{d+1}^{+}$. [20]

Theorem 1. Let $G=\left\{\gamma|d x|, x \in R^{d}\right\}$ be a space of normal distribution, where $|d x|$ is Lebesgue measurement. Then its positive definite affine space $A f f_{d}^{+}$has an explicit embedding $A f f_{d}^{+} \rightarrow S y m_{d+1}^{+}$lying on the Riemannian symmetric space $S l_{d+1} / S O_{d+1}$.

Proof. Denote an affine group of $G$ in $R^{d}: A f f_{d}=\left\{(m, Q) \mid x \rightarrow Q x+m, Q \in G_{d}, m \in\right.$

$\left.R^{d}\right\}$ acts transitively on G by $\gamma|d x| \rightarrow(m, Q) \otimes \gamma|d x|$, where $\otimes$ denotes the transitive operator.

Assume $\gamma_{0}|d x|=(2 \pi)^{-d / 2} e^{-\frac{1}{2}|x|^{2}}|d x|$ as an standard Gaussian distribution on $R^{d}$, where $\pi^{-1}\left(\gamma_{0}|d x|\right)=O_{d}$ is a positive measure. The transitive operation $(m, Q) \otimes \gamma|d x|$ can be explicitly written as

$$
(m, Q) \otimes \gamma|d x|=(2 \pi)^{-d / 2}(\operatorname{det} Q)^{-1} e^{-\frac{1}{2}\left|Q^{-1}(x-m)\right|^{2}}|d x|
$$

only when $\operatorname{det} Q>0$. Therefore, we add a restriction $\theta$ to keep affine group positive definite $\theta: A f f_{d}^{+}=\{(m, Q) \mid \operatorname{det} Q>0\}$. Since the restriction is also transitive on $G$, where $G_{d}=A f f_{d}^{+} / S O_{d}$. Considering $A f f_{d}$ as a subgroup of a larger simple Lie group $S L_{d+1}=\left\{V \mid V \in R^{d+1}\right\}$, where $A f f_{d} \subset S l_{d+1},(m, Q)$ can be embedded as

$$
A f f_{d} \rightarrow S l_{d+1},(m, Q) \rightarrow V,(m, Q) \rightarrow(\operatorname{det} Q)^{-\frac{1}{d+1}}\left(\begin{array}{cc}
Q & m \\
0 & 1
\end{array}\right)
$$

Since the simple Lie group $S l_{d+1}$ acts canonically on all symmetric definite positive matrices $S y m_{d+1}^{+}$with determinant 1. In order to meet the restriction $\theta$ of the subgroup $A f f_{d}$, the simple Lie group $S l_{d+1}$ is mapped to a symmetric definite positive space $\widetilde{\theta}: \operatorname{Sym}_{d+1}^{+}=\left\{V V^{T}\left|V V^{T} \in R^{(d+1) \times(d+1)},\right| V V^{T} \mid>0\right\}$, where

$$
S l_{d+1} \rightarrow \operatorname{Sym}_{d+1}^{+}, V \rightarrow V V^{T},(m, Q) \rightarrow(\operatorname{det} Q)^{-\frac{2}{d+1}}\left(\begin{array}{cc}
Q Q^{T}+m m^{T} & m \\
m^{T} & 1
\end{array}\right)
$$


Then the transitive action of the restriction for the simple Lie group $S l_{d+1}: G_{d+1}=$ $S l_{d+1} / S O_{d+1}$ lies on the Riemannian symmetric space with $S O_{d+1}$-principle bundle $\widetilde{\theta}: S l_{d+1} \rightarrow S y m_{d+1}^{+}, V \rightarrow V V^{T}$ Hence, Theorem 1 is proven.

In DIS, $Q Q^{T}=\widehat{C}$ Therefore, after embedding, we have 3 statistical representations:

$$
\begin{gathered}
\Phi_{S A S}=\left\{\mu \mu^{T}\left|\mu \mu^{T} \in R^{d \times d},\right| \mu \mu^{T} \mid>0\right\} \\
\Phi_{S U S}=\left\{C\left|C \in R^{d \times d},\right| C \mid>0\right\} \\
\Phi_{D I S}=\left\{V V^{T}\left|V V^{T}=\left(\begin{array}{cc}
\widehat{C}+\widehat{m} \widehat{m}^{T} & \widehat{m} \\
\widehat{m}^{T} & 1
\end{array}\right), V V^{T} \in R^{(d+1) \times(d+1)},\right| V V^{T} \mid>0\right\}
\end{gathered}
$$

125 where $\Phi_{S A S} \in S y m_{d}^{+}, \Phi_{S U S} \in S y m_{d}^{+}, \Phi_{D I S} \in S y m_{d+1}^{+}$. The SPD matrices lie in a specific Riemannian manifold, where tangent vector of $\mathrm{Sym}^{+}$is often utilized as a projection to $R^{d}$. For the three different embedded statistics and all their possible combinations, we examine logarithm Hilbert subspace through an eigen-decomposition: $\log (\boldsymbol{\Phi})=$ $U(\log (\boldsymbol{\Lambda})) U^{T}$ where $\boldsymbol{\Phi}=\left\{\Phi_{S A S}, \Phi_{S U S}, \Phi_{D I S}, \Phi_{S A S} \Phi_{S U S}, \Phi_{S A S} \Phi_{D I S}, \Phi_{S U S} \Phi_{D I S}\right.$,

$\left.{ }_{130} \Phi_{S A S} \Phi_{S U S}, \Phi_{D I S}\right\}$ indicates the set of all possible statistic combinations; $\boldsymbol{\Lambda}=\left\{\Lambda_{S A S}, \Lambda_{S U S}\right.$, $\left.\Lambda_{D I S}, \Lambda_{S A S} \Lambda_{S U S}, \Lambda_{S A S} \Lambda_{D I S}, \Lambda_{S U S} \Lambda_{D I S}, \Lambda_{S A S} \Lambda_{S U S} \Lambda_{D I S}\right\}$ indicates the set of all the possible eigen-value matrices of $\boldsymbol{\Phi}$, respectively; $U$ is the decomposed projection matrices. Set $\boldsymbol{\Phi}$ describes eigen-spaces $\boldsymbol{\Lambda}$ of all the possible statistics combinations. The last element utilizes all the statistics, while the first three have only single statistic. The log-

135 arithmic eigen-values measures the tangent subspaces of the symmetric positive definite Riemannian manifold. In the mutual subspaces method (MSM) and discriminant canonical correlations (DCC) method the diagonal eigen-matrix is also known as canonical correlations. [10] 23]

In our work, subspace with minimum deviation of the canonical correlations is selected 140 from set $\Phi$. The canonical correlation is computed as: $\cos \Theta=\operatorname{diag}(\log \boldsymbol{\Lambda})$. Subspaces are selected by $\min \{\operatorname{var}(\cos \Theta)\}$, where $\operatorname{var}(\cdot)$ calculates the deviation and $\operatorname{diag}(\cdot)$ denotes the diagonal matrix.

\subsection{Riemannian Distance Discriminant Function}

After the subspaces embedding and selection, N labelled training set $X=\left\{X_{1}, \ldots, X_{N}\right\}$ becomes $R \times N$ labelled statistical points $\boldsymbol{\Phi}=\left\{\boldsymbol{\Phi}_{\mathbf{1}}, \ldots, \boldsymbol{\Phi}_{\boldsymbol{R}}\right\}=\left\{\left\{\Phi_{1}^{1}, \ldots, \Phi_{N}^{1}\right\}, \ldots,\left\{\Phi_{1}^{R}, \ldots, \Phi_{N}^{R}\right\}\right\}$ lying on $\mathrm{Sym}^{+}$Riemannian manifolds, where $R$ is the number of selected statistics and $N$ is the number of sets for training.

In the Lie group structure, the Log-Euclidean distance (LED) for $X_{i}, X_{j} \in S_{y m}^{+}$ is defined as $\operatorname{LED}\left(X_{i}, X_{j}, t\right)=\exp \left((1-t) \log \left(X_{i}\right)+t \log \left(X_{j}\right)\right)$ where $\exp (\cdot)$ and $\log (\cdot)$ are matrix exponential and logarithm operator. Assume two Riemaninan points for $\mathrm{r}$-th statistic $\Phi_{i}^{r}, \Phi_{j}^{r}$ has two linear discriminative mapping $Y_{i}^{r}, Y_{j}^{r}$ on the domain of matrix logarithms where $\log \left(\Phi_{i}^{r}\right) Y_{i}^{r} \perp \log \left(\Phi_{j}^{r}\right) Y_{j}^{r}$, the geodesic distance between $\log \left(\Phi_{i}^{r}\right)$ and 


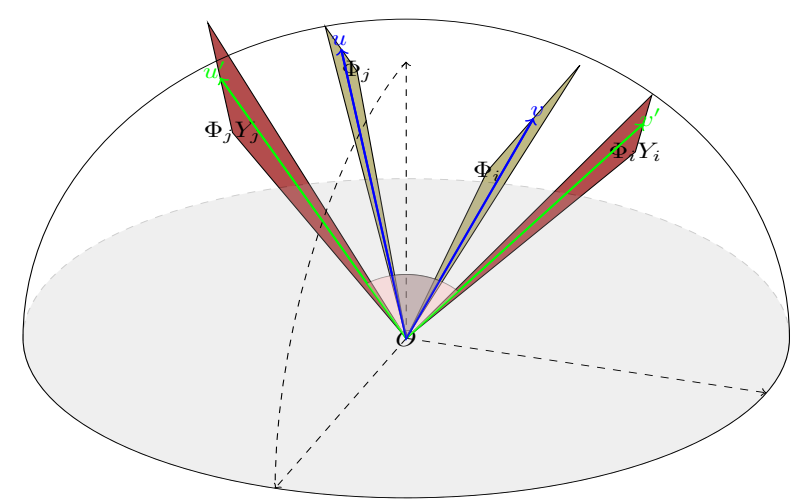

Figure 1: Mapped orthogonal subspace for a cannot-link $(i, j)$ pairs. $\Phi_{i}, \Phi_{j}$ are two Riemannian points and $\Phi_{i} Y_{i}, \Phi_{j} Y_{j}$ are Riemannian points after mapping. After mapping, $\log \left(\Phi_{i}\right) Y_{i} \perp \log \left(\Phi_{j}\right) Y_{j}$ and $\theta=$ $\angle u^{\prime} O v^{\prime}=90^{\circ}$

$\log \left(\Phi_{j}^{r}\right)$ after mapping is then computed by Euclidean distance in the domain of matrix logarithms:

$$
\begin{aligned}
d\left(\Phi_{i}^{r}, \Phi_{j}^{r}\right) & =\left\|\log \left(\Phi_{i}^{r}\right) Y_{i}^{r}-\log \left(\Phi_{j}^{r}\right) Y_{j}^{r}\right\|_{F} \\
& =\operatorname{tr}\left(\left(\log \left(\Phi_{i}^{r}\right) Y_{i}^{r}-\log \left(\Phi_{j}^{r}\right) Y_{j}^{r}\right)\left(\log \left(\Phi_{i}^{r}\right) Y_{i}^{r}-\log \left(\Phi_{j}^{r}\right) Y_{j}^{r}\right)^{T}\right)
\end{aligned}
$$

where $\|\cdot\|_{F}$ is the matrix Frobenius norm and $\operatorname{tr}(\cdot)$ is the matrix trace. Fig.1 shows the mapped subspaces graphically for a cannot-link $(i, j)$ statistic pair. As Fig.1 shows,

${ }_{150} \theta=\angle u^{\prime} O v^{\prime}$ is the principle angle. The Riemannian distance actually measures the principle angle $\|\theta\|_{2}$. If the two points are similar, the distance $d\left(\Phi_{i}^{r}, \Phi_{j}^{r}\right)=1$. If they are dissimilar, distance $d\left(\Phi_{i}^{r}, \Phi_{j}^{r}\right)=-1$. The relationship holds only when the assumed linear mapping $Y_{i}, Y_{j}$ are equally orthonormal where $Y=Y_{i}^{r}=Y_{j}^{r}$ and $Y_{i}^{r} Y_{j}^{r}=I_{d}, I_{d}$ is a $d \times d$ identity matrix.

Given a scale invariant positive definite matrix $A_{r}=Y_{i}^{r} Y_{j}^{r}$. It can be approximated with an identity matrix $A_{0}=I_{d}$ with the distance measurement $d_{A_{r}}\left(\Phi_{i}^{r}, \Phi_{j}^{r}\right)$ constrained by the must-link and cannot-link training pairs $\left(\Phi_{i}^{r}, \Phi_{j}^{r}\right)$ for the r-th statistics.

$$
d_{A_{r}}\left(\Phi_{i}^{r}, \Phi_{j}^{r}\right)=\delta_{i j} \operatorname{tr}\left(A_{r}\left(\log \left(\Phi_{i}^{r}\right)-\log \left(\Phi_{j}^{r}\right)\right)\left(\log \left(\Phi_{i}^{r}\right)-\log \left(\Phi_{j}^{r}\right)\right)^{T}\right)
$$

As a distance discriminant function, $d_{A_{r}}\left(\Phi_{i}^{r}, \Phi_{j}^{r}\right)$ shows outstanding scale invariant properties ranging from $[-1,1] . \delta_{i j}$ is an indicator function for $(i, j)$ pairs. If $(i, j)$ is mustlink, $\delta_{i j}=1$; if $(i, j)$ cannot-link $\delta_{i j}=-1$. $A_{r}$ is a Mahalanobis-like distance metric approximated with an identity matrix. After the approximation of $A_{r}$, the classification can be simply completed by nearest neighbor (NN) method using $d_{A_{r}}$ as its distance discriminant function.

\subsection{Log-Determinant Divergence Based Leaning Problem}

As discussed in Section 2.2, the learning problem is to approximate R Mahalanobislike metrics $A=\left\{A_{1}, \ldots, A_{R}\right\}$ with identity matrix $A_{0}$ for $\mathrm{R}$ selected statistics. Two 


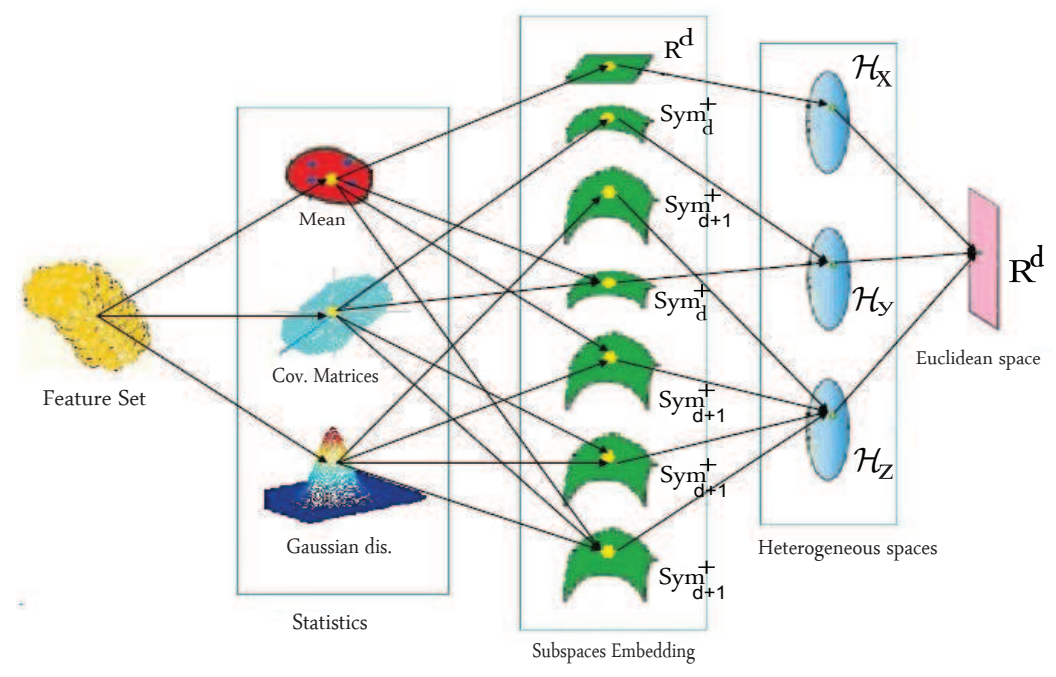

Figure 2: Data space mapping

properties must be kept during the matrix approximation: First, $A_{r}, r \in[1, R]$ must be scale invariant; Second, $A_{r}$ can be factorized as two linear mapping $A_{r}=Y_{i} Y_{j}$. Accordingly, Log-Determinant divergence is introduced as our measurement for the positive definite matrices approximation. Log-Determinant divergence is a typical measurement for positive definite matrices nearness problem proved in 24, which has also been proved to be convex and scale-invariant in 25. The optimization problem is considered as seeking $\mathrm{R}$ discriminant metrics to describe the statistical characteristics of the $N \times R$ selected statistical feature sets. Finally, our metric learning problem is expressed as

$$
\begin{array}{r}
\min _{A_{1} \leq 0, \ldots, A_{r} \leq 0, \epsilon} \frac{1}{R} \sum_{r=1}^{R} D_{l d}\left(A_{r}, A_{0}\right)+\gamma D_{l d}\left(\operatorname{diag}(\epsilon), \operatorname{diag}\left(\epsilon_{0}\right)\right) \\
\text { s.t. } \frac{\delta_{i j}}{R} \sum_{r=1}^{R} d_{A_{r}}\left(\Phi_{i}^{r}, \Phi_{j}^{r}\right) \leq \epsilon_{i j} \forall(i, j)
\end{array}
$$

${ }_{165} \delta_{i j}$ denotes the similarity between $\mathrm{i}$-th and $\mathrm{j}$-th kernel. If they are similar, $\delta_{i j}=1$, otherwise $\delta_{i j}=-1$. $D_{l d}\left(A_{r}, A_{0}\right)$ is the LogDet divergence measuring the correlation between matrix $A_{r}$ and $A_{0}$ where $D_{l d}\left(A_{r}, A_{0}\right)=\operatorname{tr}\left(A_{r}, A_{0}^{-1}\right)-\log \operatorname{det}\left(A_{r} A_{0}^{-1}\right)-d$. d is the dimension of $A . \epsilon_{i j}$ denotes the boundaries of similarity and dissimilarity, which is introduced as an upper/lower bound for the $\mathrm{i}$-th and $\mathrm{j}$-th sample covariance [26. While

170 the covariance between different classes may not always be the same, a trade-off is the self-deviation between $\epsilon$ and optimal slack $\epsilon_{0}$, which is supposed to be of unit at principle space. Thus, in order to control such a self-deviation, a second term is introduced as the trade-off correction term, where $\gamma$ is a parameter arbitrarily set before the computation. In Eq.(13), the first term of the minimization corresponds to the maximum of inter-class 175 and the second term corresponds to the minimum of intra-class.

Figure 2 gives a graphic illustration explaining the streamline of our methods. Firstly, various features set are mapped into their statistic subspaces; Secondly, by constructing the multiple statistic combinations, some combination is selected based-on its canonical correlation. Then, Riemannian distance is used as a discriminant function measuring the different statistics in the selected combination separately in the heterogeneous Hilbert space. Finally, all the statistics are measured in the Euclidean space through Mahalanobis-like identity matrices approximation with Log-Determinant divergence. 


\section{Optimizations}

The form of Eq.(9) can be considered as Bregman optimizations for R selected statistics [25, 27. With Bregman projection, Eq.(9) has the following Lagrange form:

$$
\left\{\begin{array}{l}
\nabla D_{l d}\left(A_{r}^{t+1}\right)=\nabla D_{l d}\left(A_{r}^{t}\right)+\delta_{i j} \alpha\left(\Phi_{i}-\Phi_{j}\right)^{T} A_{r} \\
\nabla D_{l d}\left(\epsilon_{i j}^{t+1}\right)=\nabla D_{l d}\left(\epsilon_{i j}^{t}\right)-\delta_{i j} \alpha / \gamma
\end{array}\right.
$$

where $\alpha$ is the Lagrange multiplier. The first and the second equation are the Bregman projection with respect to the minimizers $A_{1}, \ldots, A_{R}$ and $\epsilon$, respectively. Minimizer $A_{r}(r=1, . ., R)$ and $\epsilon$ are then iteratively updated by solving Eq.(15)

$$
\left\{\begin{array}{l}
A_{r}^{t+1}=A_{r}^{t}+\frac{\delta_{i j} \alpha}{1-\delta_{i j} \alpha d_{A_{r}^{t}}\left(\Phi_{i}^{r}, \Phi_{j}^{r}\right)} A_{r}^{t}\left(\Phi_{i}, \Phi_{j}\right)\left(\Phi_{i}, \Phi_{j}\right)^{T} A_{r}^{t} \\
\epsilon_{i j}^{t+1}=\gamma \epsilon_{i j}^{t} /\left(\gamma+\alpha \epsilon_{i j}^{t}\right)
\end{array}\right.
$$

Solution to Eq.(15) is provided in Appendix A. For solving the Lagrange multiplier, multiple inequality constrains of Eq.(13) have to be satisfied

$\frac{\delta_{i j}}{R} \sum_{r=1}^{R} \operatorname{tr}\left(A_{r}\left(\Phi_{i}^{r}, \Phi_{j}^{r}\right)\left(\Phi_{i}^{r}, \Phi_{j}^{r}\right)^{T}\right) \leq \epsilon_{i j}$ for all $(i, j)$. Thus we introduce dual variables $\gamma \geqslant 0$. By meeting the KKT condition, $\alpha$ can then be derived into a Euler-Lagrange equation with non-negative maintaining

$$
\left\{\begin{array}{l}
\frac{\delta_{i j}}{R} \sum_{r=1}^{R} d_{A_{r}^{t+1}}\left(\Phi_{i}^{r}, \Phi_{j}^{r}\right)=\epsilon_{i j}^{t+1} \\
\alpha=\min \left(\lambda_{i j}, \alpha\right), \lambda_{i j}=\lambda_{i j}-\alpha
\end{array}\right.
$$

The Lagrange multiplier $\alpha$ can be solved with Eq.(16). The proof of uniqueness and solution of $\alpha$ are provided in Appendix A. According to the optimization, statistical kernel $A_{r}(1 \leq r \leq R)$ can be learned in the following algorithm:

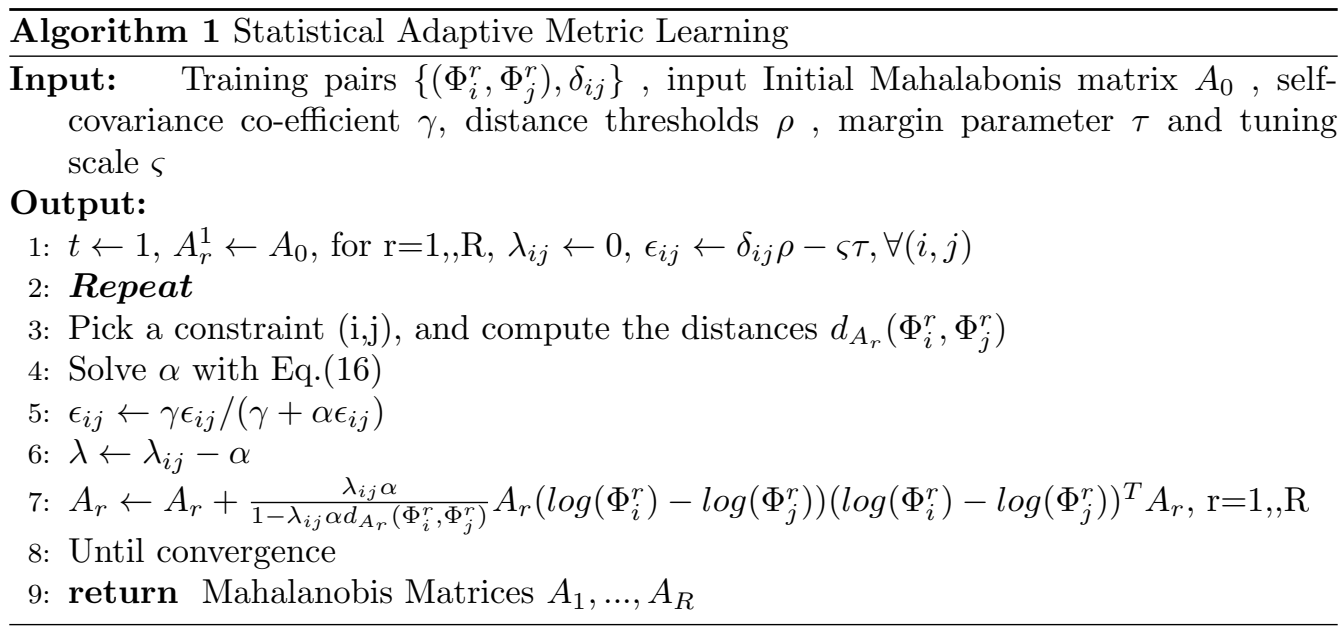




\section{Relations with Hybrid Euclidean-and-Riemannian Metric Learning (HERML) and Information Theoretic Metric Learning (ITML)}

It is essential to point out that our method, statistical adaptive metric learning (SAML) is an adaptive version of hybrid statistical metric learning, e.g. Hybrid Euclideanand-Riemannian Metric Learning (HERML). HERML individually fixes the number of learning Mahalanobis matrices as 3 for all kinds of feature sets. In HERML,

$$
\min \{\operatorname{var}(\operatorname{diag}(\log \boldsymbol{\Lambda}))\}=\log \Phi_{S A S} \Phi_{S U S} \Phi_{D I S}=U\left(\log \Lambda_{S A S} \Lambda_{S U S} \Lambda_{D I S}\right) U^{T}
$$

Eq.(13) is arbitrarily set as an implicit condition. Only full statistics combination is 190 used for their set modelling. Different from HERML, better statistic combinations are selected by analyzing the canonical correlations in SAML. Although the optimization looks similar, HERML is dealing with image sets while SAML is handling feature sets. Minor variations will usually not lead to large fluctuations in scale of images, but they may likely lead to large fluctuations in that of feature sets, e.g. HOG and SIFT. HERML individually combines all three metrics, while SAML explores the correlation among these metrics. Therefore, the subspaces scaling and effective metrics selection in SAML can achieve better performances for various feature sets.

Since our methods is described as a Mahalanobis-like metric learning, it is also essential to note that our method clearly has three advantages over ITML. Firstly, the data scale is better kept by the subspaces mapping in our method. Secondly, multiple statistic combination makes our method more adaptive to various data than that of ITML. Thirdly, the measurement of Log-determinant divergence provides a more uniformed framework for identity matrices approximation than the distribution analysis in ITML.

In ITML, a multivariate Gaussian distribution is defined directly on data as $p(x ; A)=$ $\frac{1}{Z} \exp \left(-\frac{1}{2} x^{T} A x\right)$. $\mathrm{Z}$ is the normalizing constant. The probability density $p\left(x ; A_{0}\right)$ is approximated by the initial density distribution, where $A_{0}$ is the identity matrix. In ITML, the distance between the two distributions is formulated as the KL-divergence measuring the relative entropy:

$$
K L\left(p\left(x ; A_{0}\right) \| p(x ; A)\right)=\int p\left(x ; A_{0}\right) \log \frac{p\left(x ; A_{0}\right)}{p(x ; A)} d x
$$

Then the problem identity approximation is derived as a KL-divergence minimization for $\forall(i, j)$ pairs.

$$
\min _{A \leq 0} K L\left(p\left(x ; A_{0}\right) \| p(x ; A)\right), \text { s.t. } \delta_{i j}\left(x_{i}^{T} A x_{j}\right) \leq \epsilon_{i j}, \forall(i, j)
$$

Finally, Eq.(15) is equivalent to the problem of Log-Determinant distance minimization:

$$
\min _{A \leq 0} D_{l d}\left(A, A_{0}\right)+D_{l d}\left(\operatorname{diag}(\epsilon), \operatorname{diag}\left(\epsilon_{0}\right)\right), \text { s.t. } \delta_{i j}\left(x_{i}^{T} A x_{j}\right) \leq \epsilon_{i j} \forall(i, j)
$$

where $\epsilon_{i j}$ is the slack variable for the $(i, j)$ pairs and $\epsilon_{0}$ is identity. $\delta_{i j}$ is the characteristic

Eq.(16), the minimization problem of Eq.(16) in ITML can only be used for learning single Mahalanobis metric directly on input data. The expansion of Eq.(16) is basically 
constrained by its distribution assumption in Eq.(14). Relative entropy can be computed only when all the data samples are in the same scale. Contrary to ITML, the learning problem proposed in ASML is handling multiple statistics in a uniformed minimization framework. Taking advantage of statistical subspaces embedding and log-determinant divergence, a scale invariant framework of Eq.(9) proposed in our method is capable of minimizing R Mahalanobis-like matrices and leading to better classification than that of ITML.

\section{Experiments}

\subsection{Experimental Settings}

\subsubsection{Methods in the Comparative Study}

As a statistic-based metric learning method, the proposed Statistical Adaptive Metric Learning (SAML) is mainly compared with two types of state-of-art methods in action visual feature set classification: 1) Statistic-based methods; 2) Metric learning methods. To obtain a comprehensive performance evaluation in action recognition, our method is also compared with some well-known Spatio-Temporal feature based methods.

The statistic-based methods include: 1) Sample-based methods: Maximum Mean Discrepancy (MMD) 17]; 2)Subspace-based methods: Covariance Discriminant Learning (CDL) 15 3) Distribution-based methods: Gaussian Mixture Model(GMM) 3] and Single Gaussian Model (SGM) 2].

The metric learning methods include: Information theoretic metric learning (ITML) [19, Manifold Discriminant Analysis (MDA) 14, Hybrid Euclidean-and-Riemannian Metric Learning (HERML) 4 and Distribution-based ITML (DIS-ITML).

Some well-known methods using spatio-temporal feature for action recognition were proposed by Campos et al.[28, Wang et al.[29], Fathi et al., Jhuang et al.[30, Wu et al. 31, Kovashka et al. [32, Liu et al. 33], Shao et al. 34] and Zhen et al. 35.

Static computer vision features are extracted frame by frame. In case of set modeling, common scale invariant image features are employed, including Histogram of Gradients (HOG), Shift-invariant Feature Transformation (SIFT), Harris corners and Textures. Al1 the features are clustered into 128 dimensions, while SIFT features are clustered into 128 points. Thus one frame generates a $128 \times k$ feature set. For videos with different resolutions, we uniformly resize each frame into $320 \times 240$ at QVGA resolution. When compared with other methods, feature combination of SIFT+HOG+Corners are uniform-

240 ly used. Average recognition rate is used to measure the accuracy. For statistic-based methods and metric learning methods, all the source codes of the compared methods are obtained from their original authors, except for SGM and GMM. We implemented SGM and GMM meticulously. For methods using Bregman optimization like ours, distance thresholds $\rho$ is set as the mean distances, margin parameter $\tau$ is set as the standard 245 deviations and tuning scale $\varsigma \in[0.0110]$. Furthermore, since our experiments are tested on feature sets, we carefully tuned each models of all the methods to achieve the best possible recognition performance. For MMD, the number of iteration is set to 10. For ITML, we set the parameter of Bregman optimization as stated above. The parameter settings of MDA is according to [14. For CDL we adopt the KLDA for the discriminant

250 analysis and k-NN for classification. For Spatial-Temporal (ST) feature based methods, we obtain their best recognition rate from the referred publications. 


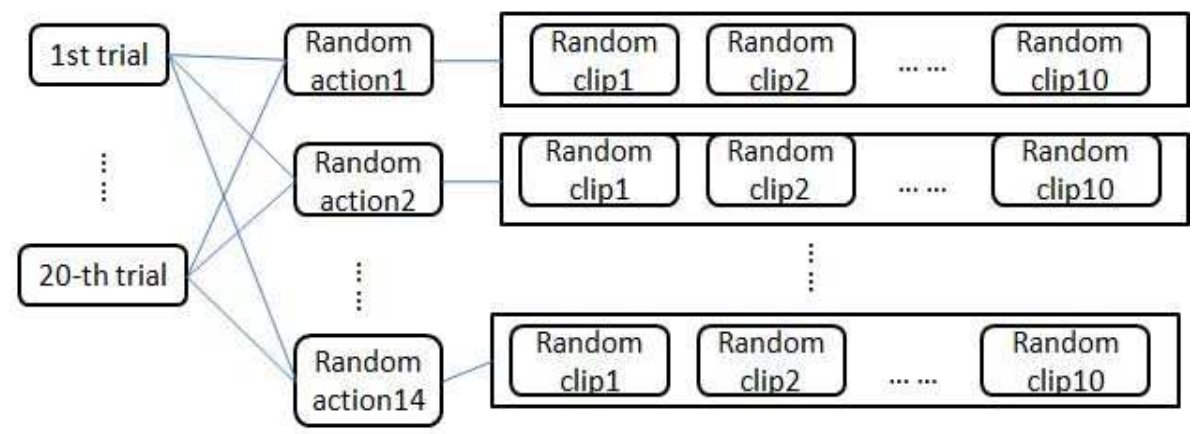

Figure 3: Illustration of sampling procedure for large action video data set

\subsubsection{Databases}

Five famous human action data sets are used in our experiments: Weizmann human action, UCF101, KTH human action, YouTube and HMDB51. Among those five video data sets, Weizmann and KTH have very static backgrounds with different human actions, while YouTube and HMDB51 both have complex and varying backgrounds and dynamic scenarios with different humans. Experiments are conducted both on human verification and action recognition.

The KTH dataset is a simple human action dataset, which includes 600 black and white videos captured by stationary cameras in 6 classes of actions with resolution of $160 * 120$. The Weizmann dataset include 10 classes of human actions with 90 videos at low resolution of $180^{*} 144$. The UCF101 human action dataset is a large dataset with 13320 videos from 101 action categories. UCF101 provides a large diversity in term of action types and with large variations in camera motion, object appearance and pose, object scale, viewpoint, cluttered background, illumination conditions, etc. It is the most challenging data set to date. The action categories can be divided into five types: 1) Human-Object Interaction 2) Body-Motion Only 3) Human-Human Interaction 4) Playing Musical Instruments 5) Sports. In our experiments we randomly select classes from all of the UCF101 categories. The YouTube dataset is a challenging and widely used video dataset. It has 1,910 video clips of 47 subjects collected from YouTube. The HMDB51 dataset was collected by Kuehne et al., containing 6,766 video clips in total. There are 51 action classes, each with at least 101 positive samples.

For smaller datasets with less than 20 classes we test on all classes. For larger datasets with more than 20 classes, we take a statistical sampling approach to evaluate the average performance. We conduct 20 trials on each of these large datasets. In each trial we randomly select 14 classes; and inside each class, we randomly select 10 video clips, 5 for training and 5 for testing. Thus 280 random action classes and 2800 random video clips are evaluated for each of these large action datasets. Average recognition rate over 20 trials is then calculated as the measure of performance. Figure 3 illustrates the sampling procedure of our experiments on large datasets. A fixed number of frames and parameter values are selected in the experiments. The training gallery includes the same number of frames for each video sequence, and the test sets are extracted in the same way. The task is to recognize the input test set based on the training gallery.

\subsection{Results and Analysis}

285 5.2.1. Performance Evaluation and Methods Comparisons

Performance comparisons are conducted with various statistic-based algorithms. Table 1 shows the performances comparison among different statistic-based methods. Three 
benchmark video analysis datasets are in use: UCF101, YouTube and HMDB51. Note that the videos sequences in those datasets are not just with human action scenes, but also with more common and complicated shapes, streets and dynamic scenarios.

From Table 1, we can observe that the sample-based statistics have clear weakness on complex scenarios. Though supported by Bregman divergence, mean vectors focusing on the centroids are far from adequate to represent the feature set. With the addition of the 2nd-order statistic, subspace-based method achieves better recognition rates. Riemannian manifold and Grassmann affine spaces scatter the data with a scalable representation. $70.3 \%$ recognition rate is achieved by covariant discriminative learning (CDL). While the distribution-based methods perform better than sample-based methods on UCF101 data set with over $80 \%$ average recognition rate, and perform similar with sample-based methods on Youtube and HDBM51 data set with a relatively low recognition rate between $25 \%$ and $35 \%$. The proposed SAML performs better than other statistic-based methods, with the highest average recognition rate on UCF101, Youtube and HMDB51 data set. Performances evaluated in Table 1 demonstrates that the proposed SAML performs better than statistic-based methods for action feature set recognition with complex background scenarios.

Table 2 shows the performances comparison among different metric learning methods. Average recognition rates are analyzed for action feature set recognition. UCF101, YouTube and HMDB51 are used. From Table 2, HERML and DIS-ITML have over $85 \%$ recognition rates on UCF101 dataset and over $70 \%$ recognition rates on YouTube dataset. The proposed SAML has better recognition performance than other metric 310 learning methods on UCF101 and HMDB51. On YouTube data, SAML performs better than other metric learning methods, except HERML. The results in Table 1 and 2 show that our method is able to adaptively take advantage of both statistic-based methods and metric learning methods, and consequently boost their performance to some extent. SAML achieves $95.5 \%$ average accuracy on UCF101 dataset, the second best performance 315 on YouTube dataset, and the best performance on HMDB51 dataset in the state-of-art statistic-based and metric learning-based methods.

\begin{tabular}{|c|c|c|c|c|}
\hline \multicolumn{2}{|c|}{ Statistic-based methods } & UCF101 & YouTube & HMDB51 \\
\hline Sample-based & MMD[17] & 73.5 & 51.2 & 32.4 \\
\hline Subspace-based & CDL[15] & 93.5 & 67.7 & 70.3 \\
\hline \multirow{2}{*}{ Distribution-based } & GMM[3] & 87.5 & 61.2 & 32.7 \\
& SGM[2] & 82.3 & 50.3 & 26.4 \\
\hline Proposed & SAML & 95.5 & 70.83 & 71.3 \\
\hline
\end{tabular}

Table 1: Average recognition rate (\%) compared with statistic-based methods on UCF101, YouTube and HMDB51 datasets.

Table 3 compares our method with some well-known Spatial-Temporal (ST) feature based action recognition methods on KTH, Weizmann and UCF101 data set. Average recognition rate is used as the measure of accuracy. From Table 3, we observe that the 320 recognition performance of SAML is better than other well-known methods. For human action recognition, the spatial-temporal features have clear advantages over large scale feature sets on modeling and optimization computation. The subspace mapping in the proposed SAML method takes advantage of scale invariant feature space and statistic 


\begin{tabular}{|c|c|c|c|}
\hline Metric learning methods & UCF101 & YouTube & HMDB51 \\
\hline ITML[19] & 76.2 & 67.6 & 51.2 \\
\hline MDA[14] & 88.9 & 65.3 & 63.3 \\
\hline HERML[4] & 92.5 & 74.6 & 68.1 \\
\hline DIS-ITML[19] & 86.1 & 70.3 & 47.8 \\
\hline SAML & 95.5 & 70.83 & 71.3 \\
\hline
\end{tabular}

Table 2: Average recognition rate (\%) compared with metric learning methods on UCF101, YouTube and HMDB51 datasets.

\begin{tabular}{|c|c|c|c|}
\hline ST-based Methods & KTH & Weizmann & UCF101 \\
\hline Campos et al.[28] & 91.5 & 96.7 & 80 \\
\hline Wang et al.[29] & 89 & 97.8 & 83.3 \\
\hline Fathi et al.[36] & 90.5 & 100 & - \\
\hline Jhuang et al.[30] & 91.7 & 98.8 & - \\
\hline Wu et al.[31] & 94.5 & - & 91.3 \\
\hline Kovashka et al.[32] & 94.5 & - & 87.3 \\
\hline Liu et al.[33] & 95 & - & - \\
\hline Shao et al.[34] & 95 & - & 93.9 \\
\hline Zhen et al.[35] & 94.5 & - & 80.7 \\
\hline SAML & 100 & 100 & 95.5 \\
\hline
\end{tabular}

Table 3: Average recognition rate (\%) compared with well-known Spatial-Temporal(ST) feature based methods on KTH, Weizmann and UCF101 human action benchmark datasets.

\begin{tabular}{|c|c|c|}
\hline ST-based Methods & HMDB51 & YouTube \\
\hline Yu et al.[37] & 46.9 & 85.2 \\
\hline Liu et al.[33] & 48.4 & 82.3 \\
\hline Shao et al.[34] & 37.3 & - \\
\hline Zhen et al.[35] & 31.7 & 80.7 \\
\hline SAML & 71.3 & 70.83 \\
\hline
\end{tabular}

Table 4: Average recognition rate (\%) compared with Spatial-Temporal(ST) feature based methods on HMDB51 and YouTube datasets. 
combination, which leads to improved recognition accuracy. Our method is further com-

pared with several ST feature based methods on HMDB51 and YouTube data set in Table 4. The proposed SAML performed $20 \%$ better than the best results from other ST feature based methods on HDMB51. But SAML does not perform well on YouTube dataset. This is because the proposed SAML is developed for subspace analysis on visual feature sets in different scales, and static visual features such as HOG, SIFT and corners 330 are adaptively combined to construct scale variant feature sets. For YouTube video data, the ST features such as HOG3D and CNN generally have very good representation already, and thus there is no clear advantage from SAML. Overall the proposed SAML has $100 \%$ accuracies on KTH and Weizmann, 95.5\% accuracy on UCF101, $71.3 \%$ accuracy on HMDB51 and 70.83\% accuracy on YouTube data set. Such results are the best on K-

335 TH, Weizmann, UCF101, HMDB51, and the second best on YouTube. In summary, the proposed SAML outperforms most of the state-of-art methods, including statistic-based methods, metric learning methods and ST feature-based methods, on five benchmark databases.

Furthermore, as a hybrid statistical metric learning, the proposed SAML is compared 340 with other hybrid methods, i.e. HERML and DIS-ITML, in term of class-based classification accuracy. 14 action classes were randomly chosen from UCF101. For each class we randomly sampled 10 actions, 5 for training and 5 for testing. The average recognition rate is computed by averaging 10 trials of sampling. Figure 4 shows the average classification accuracy over 14 randomly sampled action classes. For 14 classes with dif-

345 ferent statistic characters, the recognition rates of SAML is higher than that of others. More importantly, no class is classified with extremely low recognition rate. Such a result demonstrates that our method is robust to various kinds of action sets. In another words, SAML is more stable than other hybrid statistical metric learning methods, and would provide similar level of recognition rate uniformly over different human action types.

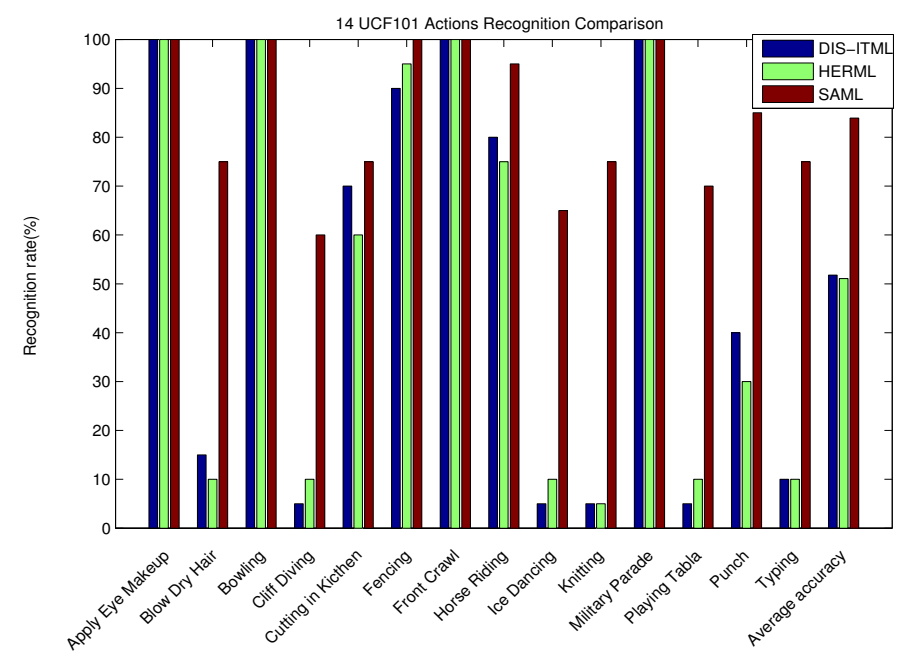

Figure 4: Classification comparison on UCF101 dataset among DIS-ITML, HERML and SAML in term of average class recognition rate(\%). The last column show an average recognition rate over all the classes. 


\subsubsection{Performance Analysis}

According to the experimental setup, two parameters of the algorithm and one variable of the video clips are to be evaluated. They are tuning scale $\varsigma$, slack weights $\gamma$ and frames in use for each video, respectively. In desirable circumstance, the classification performance should be robust to algorithm parameters. If more frames are used, which are considered as additional information, the recognition rate should also increase.

Figure 5 compares the robustness of tuning scale $\varsigma$, slack weights $\gamma$ in SAML and that of HERML, which has similar optimization and parameter settings in the algorithm design. 5 human action classes in UCF dataset were tested for recognition accuracy in this experiment. Average recognition rate is calculated on each value of the parameter. From Figure 5, we observe that the recognition rate of SAML becomes stable when zeta is no less than 0.3 and slack weights are no less than 0.1, so as the HERML. To sum up, both HERML and SAML have the similar level of parameter robustness.

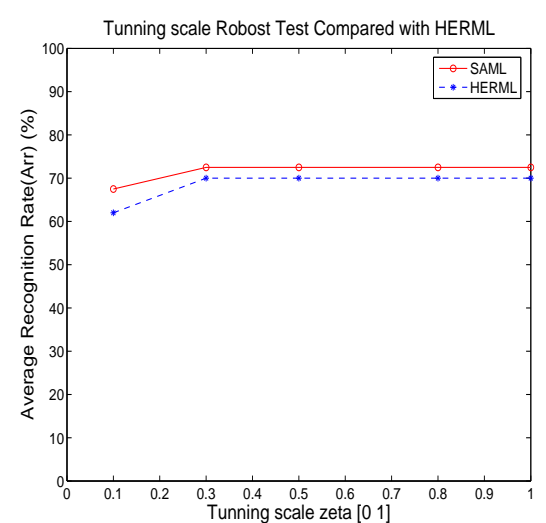

(a)

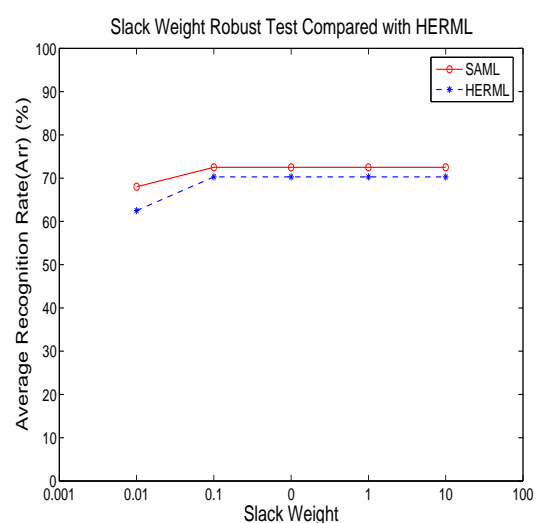

(b)

Figure 5: Evaluation of the parameter robustness with SAML and HERML. The red solid line marked by circles is the average recognition rate $(\%) /$ parameter of SAML and blue dashed line marked by asterisks is the average recognition rate (\%)/parameter of HERML. The recognition is conducted on 5 different action classes in UCF dataset. (a) Tuning scale zeta; (b) Slack weights.

Other than parameter robust evaluation, an evaluation of frames robustness is given by Figure 6 in term of average recognition rate w.r.t. number of frames. Three hybrid statistic metric learning methods are evaluated on three datasets: KTH, Weizmann and HMDB51. From Figure 6, we observe that all the recognition rates are increasing among all three of the data set as the number of frames increases with SAML and HERML, while the classification rates of DIS-ITML on HMDB51 and Weizmann remain the same. Therefore in the case of feature set classification, our method and HERML are robust to the number of frames while DIS-ITML is not. In addition, Figure 7 compares the average recognition rate w.r.t. number of frames among all the three hybrid statistic methods. Not only robust to frames, SAML also performs the best in this case. 


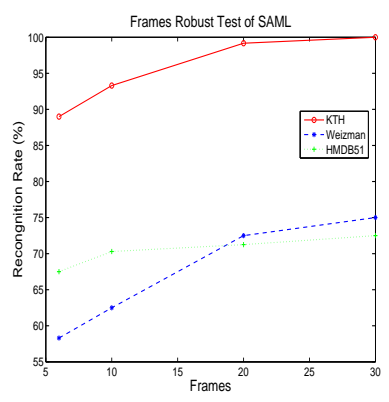

(a)

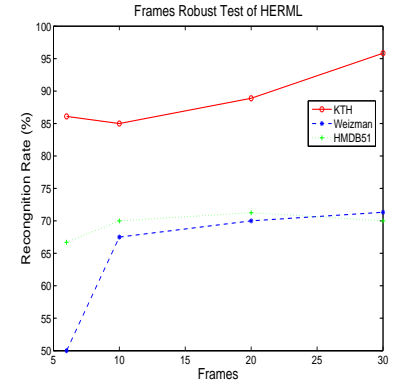

(b)

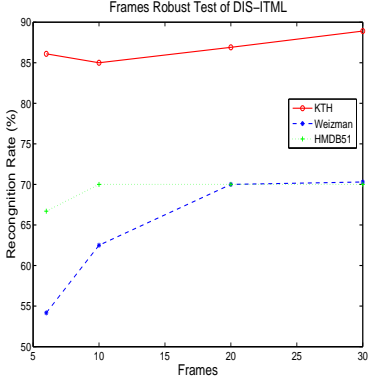

(c)

Figure 6: Evaluation of the frame robustness with hybrid statistical methods. The red solid line marked by circles is the recognition rate (\%) on KTH data; the blue dotted line marked by asterisks is the recognition rate (\%) on Weizmann data and the green dashed line marked with plus signs is the recognition rate (\%) on HMDB51 data. (a) SAML; (b) HERML; (c) DIS-ITML

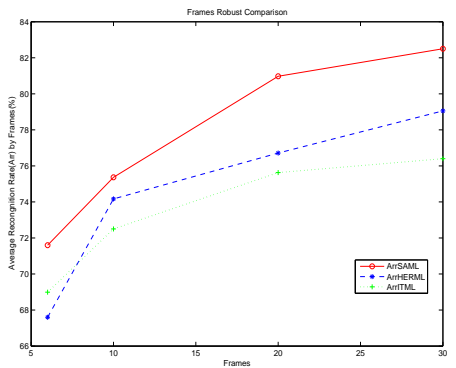

Figure 7: Comparison of recognition rate with different number of frames among three hybrid statistic methods. The red solid line marked by circles is the average recognition rate (Arr) (\%) of SAML; the blue dotted line marked by asterisks is the recognition rate (\%) of HERML and the green dashed line marked with plus sign is the recognition rate $(\%)$ of DIS-ITML. The recognition is conducted on the human recognition for 5 different classes on UCF dataset. 
Furthermore, we test our method on different feature combinations to evaluate the robustness of various features. Figure 8 shows an example of recognition rate with 14 randomly actions classes from Youtube database. With 5 available static features, the figure shows that the recognition rates of SAML are nearly the same among different combinations for each of the actions.

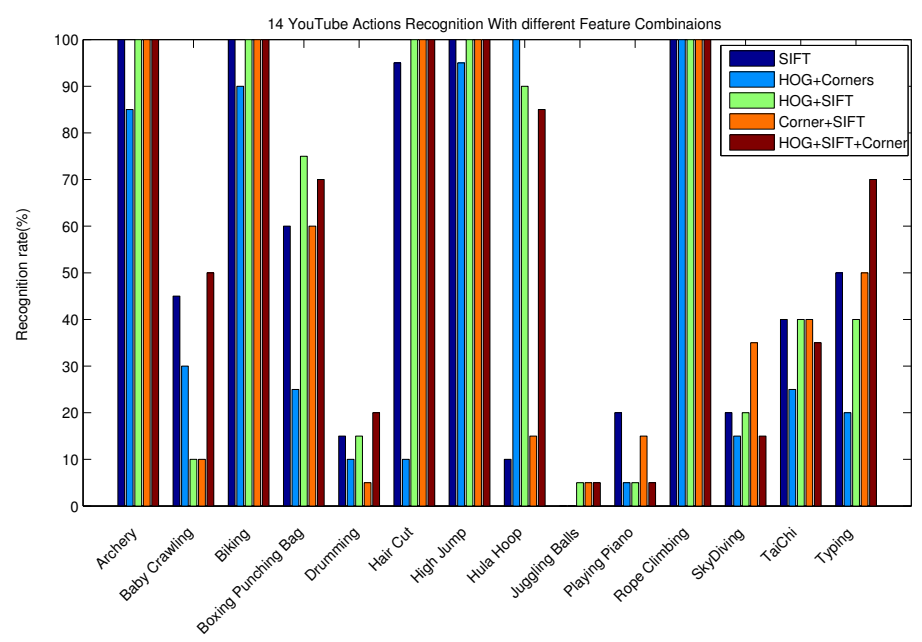

Figure 8: Robustness test of SAML with different feature combinations on YouTube database in terms of recognition rate $(\%)$. Feature combinations: SIFT, HOG+Corners, HOG+SIFT, Corner+SIFT, HOG+SIFT+Corner

\subsubsection{Computational Costs}

Finally, computational costs of our method on different data sets are also presented in Table 4 and Table 5. In comparison, the computational cost of HERML is provided as well. All the classifications were set up as stated in section 5.1. From Table 4 and 5, we observe that both the training time and testing time of the proposed SAML are faster than those of HERML. Such acceleration is mainly due to the statistic kernel reduction introduced by our minimum Hilbert subspace selection. Classifications with only necessary statistics combination efficiently improve the computation and recognition.

Table 5: Gallery training time (in seconds) of HERML and SAML in the experiments on five video databases: UCF101, KTH, Weizmann, YouTube and HMDB51.

\begin{tabular}{|c|c|c|c|c|c|}
\hline Training time & UCF101 & KTH & Weizmann & YouTube & HMDB51 \\
\hline HERML & 227.2 & 765.737 & 280.57 & 366.99 & 567.36 \\
\hline SAML & 77.43 & 123.676 & 71.35 & 100.77 & 83.49 \\
\hline
\end{tabular}

In summary, our method performs well in human action recognition. It generally outperforms other hybrid statistic metric learning methods in terms of parameter robustness and computational efficiency. 
Table 6: Recognition test time (in seconds) of HERML and SAML in the experiments on five video databases: UCF101, KTH, Weizmann, YouTube and HMDB51.

\begin{tabular}{|c|c|c|c|c|c|}
\hline Testing time & UCF101 & KTH & Weizmann & YouTube & HMDB51 \\
\hline HERML & 0.03 & 0.037 & 0.0351 & 0.037 & 0.0342 \\
\hline SAML & 0.0277 & 0.031 & 0.029 & 0.032 & 0.0335 \\
\hline
\end{tabular}

\section{Conclusions}

In this paper, we proposed a statistical adaptive metric learning method for feature set modeling and classification. The extensive experiments have shown that our proposed method outperforms several state-of-the-art methods in human action recognition over a variety of public data sets. To our best knowledge, the optimal selection of metric learning combination in the Hilbert spaces has not been investigated before this work. In the future, it would be interesting to expand our method for other possible hybrid

395 metric selections with more robust statistics modeling feature sets in different structures and real-world classifications.

\section{Acknowledgment}

This work was supported in part by the National Science Foundation under grant 1350763.

\section{Appendix A Solution to Bregman Optimization}

Consider the following constrained minimization

$$
\begin{aligned}
& \min _{A_{1} \leq 0, \ldots, A_{R} \leq 0, \epsilon} \frac{1}{R} \sum_{r=1}^{R} D_{l d}\left(A_{r}, A_{0}\right)+\gamma D_{l d}\left(\operatorname{diag}(\epsilon), \operatorname{diag}\left(\epsilon_{0}\right)\right) \\
& \text { s.t. } \quad \frac{\delta_{i j}}{R} \sum_{r=1}^{R} d_{A_{r}}\left(\Phi_{i}^{r}, \Phi_{j}^{r}\right) \leq \epsilon_{i j} \forall(i, j)
\end{aligned}
$$

where $A_{r}(r=1, R)$ is additive separable for all $\mathrm{R}$ matrices. Simplify the minimization as

$$
\begin{aligned}
& \min _{A \leq 0, \epsilon} D_{l d}\left(A, A_{0}\right)+\gamma D_{l d}\left(\operatorname{diag}(\epsilon), \operatorname{diag}\left(\epsilon_{0}\right)\right) \\
& \text { s.t. } \quad \frac{\delta_{i j}}{R} d_{A}\left(\Phi_{i}, \Phi_{j}\right) \leq \epsilon_{i j} \forall(i, j)
\end{aligned}
$$

where $d_{A}\left(\Phi_{i}, \Phi_{j}\right)=\operatorname{tr}\left(A\left(\log \left(\Phi_{i}\right)-\log \left(\Phi_{j}\right)\right)\left(\log \left(\Phi_{i}\right)-\log \left(\Phi_{j}\right)\right)^{T}\right)$. The Mahalabonis distance matrix $\mathrm{A}$ is the primal minimizer.

Firstly, with Bregman projection with respect to A,

$$
\nabla D_{l d}\left(A^{t+1}\right)=\nabla D_{l d}\left(A^{t}\right)+\delta_{i j} \alpha\left(\log \left(\Phi_{i}\right)-\log \left(\Phi_{j}\right)\right)\left(\log \left(\Phi_{i}\right)-\log \left(\Phi_{j}\right)\right)^{T} A^{t}
$$


where $\alpha$ is the Lagrange multiplier, since

$$
\nabla D_{l d}(A)=\nabla_{A}\left(\operatorname{tr}\left(A A_{0}^{-1}\right)\right)-\nabla_{A}\left(\log \operatorname{det}\left(A A_{0}^{-1}\right)\right)=A_{0}^{-1}-\frac{\left(A A_{0}^{-1}\right)^{*}}{\operatorname{det} A A_{0}^{-1}} A_{0}^{-1}=A_{0}^{-1}-A^{-1}
$$

Eq.(19) is derived to

$$
\begin{aligned}
& \left(A^{t+1}\right)^{-1}=\left(A^{t}\right)^{-1}-\delta_{i j} \alpha\left(\log \left(\Phi_{i}\right)-\log \left(\Phi_{j}\right)\right)\left(\log \left(\Phi_{i}\right)-\log \left(\Phi_{j}\right)\right)^{T} \\
& A^{t+1}=\left(\left(A^{t}\right)^{-1}-\delta_{i j} \alpha\left(\log \left(\Phi_{i}\right)-\log \left(\Phi_{j}\right)\right)\left(\log \left(\Phi_{i}\right)-\log \left(\Phi_{j}\right)\right)^{T}\right)^{-1}
\end{aligned}
$$

According to the Sherman-Morris inverse formula:

$$
\left(B+u v^{T}\right)^{-1}=B^{-1}-\frac{B^{-1} u v^{T} B^{-1}}{1+v^{T} B^{-1} u}
$$

where $\mathrm{u}, \mathrm{v}$ is semi-positive definite matrix and $\mathrm{B}$ is symmetric positive definite (SPD) matrix. Eq.(22) is then derived in form of

$$
A^{t+1}=A^{t}+\frac{\delta_{i j} \alpha A^{t}\left(\log \left(\Phi_{i}\right)-\log \left(\Phi_{j}\right)\right)\left(\log \left(\Phi_{i}\right)-\log \left(\Phi_{j}\right)\right)^{T} A^{t}}{1-\left(\log \left(\Phi_{i}\right)-\log \left(\Phi_{j}\right)\right)^{T} A^{t}\left(\log \left(\Phi_{i}\right)-\log \left(\Phi_{j}\right)\right)}
$$

Simplify Eq.(24) as

$$
\begin{gathered}
A^{t+1}=A^{t}+\frac{\delta_{i j} \alpha}{1-\delta_{i j} \alpha \operatorname{tr}\left(A^{t}\left(\log \left(\Phi_{i}\right)-\log \left(\Phi_{j}\right)\right)\left(\log \left(\Phi_{i}\right)-\log \left(\Phi_{j}\right)\right)^{T}\right)} \\
\cdot A^{t}\left(\log \left(\Phi_{i}\right)-\log \left(\Phi_{j}\right)\right)\left(\log \left(\Phi_{i}\right)-\log \left(\Phi_{j}\right)\right)^{T} A^{t} \\
A^{t+1}=A^{t}+\frac{\delta_{i j} \alpha}{1-\delta_{i j} \alpha d_{A^{t}}\left(\log \left(\Phi_{i}\right)-\log \left(\Phi_{j}\right)\right)} A^{t}\left(\log \left(\Phi_{i}\right)-\log \left(\Phi_{j}\right)\right)\left(\log \left(\Phi_{i}\right)-\log \left(\Phi_{j}\right)\right)^{T} A^{t}
\end{gathered}
$$

The primal minimizer A is solved by Eq.(26). Secondly, after solving primal minimizer, look back at Eq.(18) for the solution of the other minimizer. With Bregman projection of Eq.(18) with respect to $\epsilon$,

$$
\begin{gathered}
\nabla D_{l d}\left(\epsilon_{i j}^{t+1}\right)=\nabla D_{l d}\left(\epsilon_{i j}^{t}\right)-\frac{\delta_{i j} \alpha}{\gamma} \\
\nabla D_{l d}(\epsilon)=\nabla_{\epsilon}\left[\operatorname{tr}\left(\operatorname{diag}(\epsilon) \cdot \operatorname{diag}\left(\epsilon_{0}\right)^{-1}\right)\right]-\nabla_{\epsilon}\left[\log \operatorname{det}(\operatorname{diag}(\epsilon)) \cdot \operatorname{diag}\left(\epsilon_{0}\right)^{-1}\right] \\
=\operatorname{diag}\left(\epsilon_{0}\right)^{-1}-\operatorname{diag}(\epsilon)^{-1}
\end{gathered}
$$


Then Eq.(27) becomes

$$
\begin{aligned}
\operatorname{diag}\left(\epsilon_{i j}^{t+1}\right) & =\left(\operatorname{diag}\left(\epsilon_{i j}^{t}\right)^{-1}-\frac{\delta_{i j} \alpha}{\gamma}\right)^{-1} \\
& =\operatorname{diag}\left(\epsilon_{i j}^{t}\right)+\frac{\delta_{i j} \alpha \cdot \operatorname{diag}\left(\epsilon_{i j}^{t}\right) \cdot \operatorname{diag}\left(\epsilon_{i j}^{t}\right)}{\gamma-\delta_{i j} \alpha \operatorname{diag}\left(\epsilon_{i j}^{t}\right)}=\frac{\gamma \operatorname{diag}\left(\epsilon_{i j}^{t}\right)}{\gamma-\delta_{i j} \alpha \operatorname{diag}\left(\epsilon_{i j}^{t}\right)} \\
\Rightarrow \epsilon_{i j}^{t+1} & =\frac{\gamma \epsilon_{i j}^{t}}{\gamma-\delta_{i j} \alpha \epsilon_{i j}^{t}}
\end{aligned}
$$

The updating of two minimizers $\epsilon$ and $A$ can be re-written together as

$$
\begin{cases}A^{t+1} & =A^{t}+\frac{\delta_{i j} \alpha}{1-\delta_{i j} \alpha d_{A^{t}}\left(\Phi_{i}, \Phi_{j}\right)} A^{t}\left(\log \left(\Phi_{i}\right)-\log \left(\Phi_{j}\right)\right)\left(\log \left(\Phi_{i}\right)-\log \left(\Phi_{j}\right)\right)^{T} A^{t} \\ \epsilon_{i j}^{t+1} & =\frac{\gamma \epsilon_{i j}}{\gamma-\delta_{i j} \alpha \epsilon_{i j}^{t}}\end{cases}
$$

Finally, given the updated minimizers, Lagrange multiplier $\alpha$ can be solved by the following Euler-Lagrange equation with a non-negative maintaining equation for $\alpha$ handling the inequality constrains.

$$
\left\{\begin{array}{l}
\delta_{i j} \operatorname{tr}\left(A^{t+1}(\alpha)\left(\log \left(\Phi_{i}\right)-\log \left(\Phi_{j}\right)\right)\left(\log \left(\Phi_{i}\right)-\log \left(\Phi_{j}\right)\right)^{T}\right)-\epsilon_{i j}^{t}(\alpha)=0 \\
\alpha=\min \left(\lambda_{i j}, \alpha\right)=\lambda_{i j}-\alpha
\end{array}\right.
$$

where $\alpha$ is the Lagrange multiplier, $r=1, \ldots, R$, dual variable $\lambda_{i j} \leq 0$. Note that for semi-positive definite matrix $\mathrm{z}$ and $\mathrm{SPD}$ matrix $\mathrm{B}, \operatorname{tr}\left(B z z^{T}\right)=z^{T} B z$. Thus, according to Eq.(31)

$$
\begin{aligned}
& \delta_{i j}\left[d_{A^{t}}\left(\Phi_{i}, \Phi_{j}\right)+\frac{\alpha \delta_{i j} d_{A^{t}}\left(\Phi_{i}, \Phi_{j}\right) d_{A^{t}}\left(\Phi_{i}, \Phi_{j}\right)}{1-\alpha \delta_{i j} d_{A^{t}}\left(\Phi_{i}, \Phi_{j}\right)}\right]=\frac{\gamma \epsilon_{i j}^{t}}{\gamma-\alpha \delta_{i j} \epsilon_{i j}^{t}} \\
& \Rightarrow \delta_{i j} \frac{d_{A^{t}}\left(\Phi_{i}, \Phi_{j}\right)}{1-\alpha \delta_{i j} d_{A^{t}}\left(\Phi_{i}, \Phi_{j}\right)}=\frac{\gamma \epsilon_{i j}^{t}}{\gamma-\alpha \delta_{i j} \epsilon_{i j}^{t}}
\end{aligned}
$$

Since all the elements in $d_{A^{t}}\left(\Phi_{i}, \Phi_{j}\right)=\operatorname{tr}\left(A\left(\Phi_{i}-\Phi_{j}\right)\left(\Phi_{i}-\Phi_{j}\right)^{T}\right)$ are either semi-positive definite or positive definite, $d_{A^{t}}>0$. By adding the dual variables $\lambda_{i j}, \epsilon>0$. Therefore, with all these condition above, we can conclude that a unique solution of $\alpha$ exists. The Lagrange multiplier can be solved by Eq.(32).

In summary, the simplified single statistic minimization of Eq.(18) is solved by Eq.(30) and Eq.(32).For $r$-th $(r=1,, R)$ statistic, the solution of the minimizers can then be easily written as

$$
\begin{cases}A_{r}^{t+1} & =A_{r}^{t}+\frac{\delta_{i} j \alpha}{1-\delta_{i j} \alpha d_{A_{r}^{t}}\left(\Phi_{i}^{r}, \Phi_{j}^{r}\right)} A_{r}^{t}\left(\log \left(\Phi_{i}^{r}\right)-\log \left(\Phi_{j}^{r}\right)\right)\left(\log \left(\Phi_{i}^{r}\right)-\log \left(\Phi_{j}^{r}\right)\right)^{T} A_{r}^{t} \\ \epsilon_{i j}^{t+1} & =\frac{\gamma \epsilon_{i j}^{t}}{\gamma-\delta_{i j} \alpha \epsilon_{i j}^{t}}\end{cases}
$$

With the Lagrange multiplier solved by

$$
d_{A_{r}^{t}}\left(\Phi_{i}^{r}, \Phi_{j}^{r}\right)+\frac{\delta_{i j}}{R} \sum_{r=1}^{R} \frac{\alpha d_{A_{r}^{t}}\left(\Phi_{i}^{r}, \Phi_{j}^{r}\right)}{1-\alpha d_{A_{r}^{t}}\left(\Phi_{i}^{r}, \Phi_{j}^{r}\right)}=\frac{\gamma \epsilon_{i j}^{t}}{\gamma-\alpha \delta_{i j} \epsilon_{i j}^{t}}
$$




\section{References}

[1] Q. Cai, Y. Yin, H. Man, Learning spatio-temporal dependencies for action recognition, in: Image Processing (ICIP), 2013 20th IEEE International Conference on, IEEE, 2013, pp. 3740-3744.

[2] M. Tistarelli, E. Grosso, Identity management in face recognition systems, in: Biometrics and Identity Management, Springer, 2008, pp. 67-81.

[3] O. Arandjelović, G. Shakhnarovich, J. Fisher, R. Cipolla, T. Darrell, Face recognition with image sets using manifold density divergence, in: Computer Vision and Pattern Recognition, 2005. CVPR 2005. IEEE Computer Society Conference on, Vol. 1, IEEE, 2005, pp. 581-588.

420 [4] Z. Huang, R. Wang, S. Shan, X. Chen, Hybrid euclidean-and-riemannian metric learning for image set classification, in: Computer Vision-ACCV 2014, Springer, 2015, pp. 562-577.

[5] Z. Huang, R. Wang, S. Shan, X. Chen, Learning euclidean-to-riemannian metric for point-to-set classification, in: Computer Vision and Pattern Recognition (CVPR), 2014 IEEE Conference on, IEEE, 2014, pp. 1677-1684.

[6] Z. Huang, X. Zhao, S. Shan, R. Wang, X. Chen, Coupling alignments with recognition for stillto-video face recognition, in: Computer Vision (ICCV), 2013 IEEE International Conference on, IEEE, 2013, pp. 3296-3303.

[7] Y. Hu, A. S. Mian, R. Owens, Sparse approximated nearest points for image set classification, in: Computer vision and pattern recognition (CVPR), 2011 IEEE conference on, IEEE, 2011, pp. $121-128$.

[8] M. Yang, P. Zhu, L. Van Gool, L. Zhang, Face recognition based on regularized nearest points between image sets, in: Automatic Face and Gesture Recognition (FG), 2013 10th IEEE International Conference and Workshops on, IEEE, 2013, pp. 1-7.

[9] P. Zhu, L. Zhang, W. Zuo, D. Zhang, From point to set: Extend the learning of distance metrics, in: Computer Vision (ICCV), 2013 IEEE International Conference on, IEEE, 2013, pp. 2664-2671.

[10] T.-K. Kim, J. Kittler, R. Cipolla, Discriminative learning and recognition of image set classes using canonical correlations, Pattern Analysis and Machine Intelligence, IEEE Transactions on 29 (6) (2007) 1005-1018.

[11] O. Yamaguchi, K. Fukui, K.-i. Maeda, Face recognition using temporal image sequence, in: Automatic Face and Gesture Recognition, 1998. Proceedings. Third IEEE International Conference on, IEEE, 1998, pp. 318-323.

[12] R. Wang, S. Shan, X. Chen, Q. Dai, W. Gao, Manifold-manifold distance and its application to face recognition with image sets, Image Processing, IEEE Transactions on 21 (10) (2012) 4466-4479.

[13] J. Hamm, D. D. Lee, Grassmann discriminant analysis: a unifying view on subspace-based learning, in: Proceedings of the 25th international conference on Machine learning, ACM, 2008, pp. 376-383.

[14] R. Wang, X. Chen, Manifold discriminant analysis, in: Computer Vision and Pattern Recognition, 2009. CVPR 2009. IEEE Conference on, IEEE, 2009, pp. 429-436.

[15] R. Wang, H. Guo, L. S. Davis, Q. Dai, Covariance discriminative learning: A natural and efficient approach to image set classification, in: Computer Vision and Pattern Recognition (CVPR), 2012 IEEE Conference on, IEEE, 2012, pp. 2496-2503.

[16] J. Lu, G. Wang, P. Moulin, Image set classification using holistic multiple order statistics features and localized multi-kernel metric learning, in: Computer Vision (ICCV), 2013 IEEE International Conference on, IEEE, 2013, pp. 329-336.

[17] A. Gretton, K. M. Borgwardt, M. J. Rasch, B. Schölkopf, A. Smola, A kernel two-sample test, The Journal of Machine Learning Research 13 (1) (2012) 723-773.

[18] H. Cevikalp, B. Triggs, Face recognition based on image sets, in: Computer Vision and Pattern Recognition (CVPR), 2010 IEEE Conference on, IEEE, 2010, pp. 2567-2573.

[19] J. V. Davis, B. Kulis, P. Jain, S. Sra, I. S. Dhillon, Information-theoretic metric learning, in: Proceedings of the 24th international conference on Machine learning, ACM, 2007, pp. 209-216.

[20] S.-i. Amari, H. Nagaoka, Methods of information geometry, Vol. 191, American Mathematical Soc., 2007.

[21] Z. Huang, R. Wang, S. Shan, X. Chen, Face recognition on large-scale video in the wild with hybrid euclidean-and-riemannian metric learning, Pattern Recognition.

[22] L. Shao, L. Liu, M. Yu, Kernelized multiview projection for robust action recognition, International Journal of Computer Vision (2015) 1-15.

[23] K. Fukui, O. Yamaguchi, Face recognition using multi-viewpoint patterns for robot vision, in: Robotics Research. The Eleventh International Symposium, Springer, 2005, pp. 192-201.

[24] I. S. Dhillon, J. A. Tropp, Matrix nearness problems with bregman divergences, SIAM Journal on Matrix Analysis and Applications 29 (4) (2007) 1120-1146. 
470 [25] B. Kulis, M. A. Sustik, I. S. Dhillon, Low-rank kernel learning with bregman matrix divergences, The Journal of Machine Learning Research 10 (2009) 341-376.

[26] L. M. Bregman, The relaxation method of finding the common point of convex sets and its application to the solution of problems in convex programming, USSR computational mathematics and mathematical physics 7 (3) (1967) 200-217.

27 B. Kulis, M. Sustik, I. Dhillon, Learning low-rank kernel matrices, in: Proceedings of the 23rd international conference on Machine learning, ACM, 2006, pp. 505-512.

[28] T. De Campos, M. Barnard, K. Mikolajczyk, J. Kittler, F. Yan, W. Christmas, D. Windridge, An evaluation of bags-of-words and spatio-temporal shapes for action recognition, in: Applications of Computer Vision (WACV), 2011 IEEE Workshop on, IEEE, 2011, pp. 344-351.

[29] Z.-J. Wang, Y.-G. Chen, Logarithmic least squares prioritization and completion methods for interval fuzzy preference relations based on geometric transitivity, Information Sciences 289 (2014) 59-75.

[30] H. Jhuang, T. Serre, L. Wolf, T. Poggio, A biologically inspired system for action recognition, in: Computer Vision, 2007. ICCV 2007. IEEE 11th International Conference on, Ieee, 2007, pp. 1-8.

[31] X. Wu, D. Xu, L. Duan, J. Luo, Action recognition using context and appearance distribution features, in: Computer Vision and Pattern Recognition (CVPR), 2011 IEEE Conference on, IEEE, 2011, pp. 489-496.

[32] A. Kovashka, K. Grauman, Learning a hierarchy of discriminative space-time neighborhood features for human action recognition, in: Computer Vision and Pattern Recognition (CVPR), 2010 IEEE Conference on, IEEE, 2010, pp. 2046-2053.

[33] L. Liu, L. Shao, X. Li, K. Lu, Learning spatio-temporal representations for action recognition: A genetic programming approach, Cybernetics, IEEE Transactions on 46 (1) (2016) 158-170.

[34] L. Shao, X. Zhen, D. Tao, X. Li, Spatio-temporal laplacian pyramid coding for action recognition, Cybernetics, IEEE Transactions on 44 (6) (2014) 817-827.

495 [35] X. Zhen, L. Shao, X. Li, Action recognition by spatio-temporal oriented energies, Information Sciences 281 (2014) 295-309.

[36] A. Fathi, G. Mori, Action recognition by learning mid-level motion features, in: Computer Vision and Pattern Recognition, 2008. CVPR 2008. IEEE Conference on, IEEE, 2008, pp. 1-8.

[37] M. Yu, L. Liu, L. Shao, Structure-preserving binary representations for rgb-d action recognition. 
